\title{
ORFEES - a radio spectrograph for the study of solar radio bursts and space weather applications
}

\author{
Abdallah Hamini ${ }^{1,2}$, Gabriel Auxepaules ${ }^{2}$, Lionel Birée ${ }^{3}$, Guy Kenfack ${ }^{2}$, Alain Kerdraon ${ }^{1}$, \\ Karl-Ludwig Klein ${ }^{1,2, *}$, Patrice Lespagnol ${ }^{2}$, Sophie Masson ${ }^{1,2}$, Lucile Coutouly ${ }^{2}$, \\ Christian Fabrice ${ }^{2}$, and Renaud Romagnan ${ }^{1}$ \\ ${ }^{1}$ Observatoire de Paris, LESIA, Univ. PSL, CNRS, Sorbonne Univ., Univ. de Paris, 5 Place Jules Janssen, 92190 Meudon, France \\ 2 Station de radioastronomie de Nançay, Univ. PSL, CNRS, Univ. d'Orléans, 18330 Nançay, France \\ ${ }^{3}$ Commandement de l'Espace/Centre Opérationnel de Surveillance Militaire des Objets Spatiaux, Base Aérienne 942, BP19, \\ 69579 Limonest Cedex, France
}

Received 27 May 2021 / Accepted 12 October 2021

\begin{abstract}
Radio bursts are sensitive tracers of non-thermal electron populations in the solar corona. They are produced by electron beams and shock waves propagating through the corona and the heliosphere, and by trapped electron populations in coronal mass ejections (CMEs) and in quiescent active regions. Combining space-borne and ground-based radio spectrographs allows one to track disturbances between the low corona, near or at the sites of particle acceleration, and the spacecraft. Radio observations are, therefore, a significant tool in probing the solar origin of heliospheric disturbances, which is a central research topic as witnessed by the Parker Solar Probe and Solar Orbiter missions. The full scientific return of these projects needs vigorous ground-based support, which at radio wavelengths covers altitudes up to about a solar radius above the photosphere. Besides research in solar and heliospheric physics, monitoring solar radio bursts also supports space weather services. On occasion, radio bursts can themselves be a space weather hazard. The Nançay radio astronomy station in central France has a long tradition of monitoring radio emission at decimetre-to-meter wavelengths. This article describes the radio spectrograph ORFEES (Observations Radiospectrographiques pour FEDOME et l'Etude des Eruptions Solaires). It observes the whole-Sun flux density between 144 and $1004 \mathrm{MHz}$, pertaining to regions between the low corona and about half a solar radius above the photosphere. ORFEES results from a partnership between Observatoire de Paris and the French Air Force, which operates the experimental space weather service FEDOME. The primary use of the instrument at the Paris Observatory is astrophysical observation. Low-resolution data with rapid availability are presently produced for the French Air Force. Similar information can be made available to a broader range of space weather service providers. This article gives an overview of the instrument design and access to the data and shows a few illustrative observations.
\end{abstract}

Keywords: Astronomical instrumentation / methods and techniques / Sun: activity / Sun: corona / Sun: coronal mass ejections (CMEs) / Sun: radio radiation

\section{Introduction}

Radio bursts are signatures of explosive energy release in the solar corona related to flares and coronal mass ejections (CMEs). The bursts are emitted by electrons at suprathermal to relativistic energies. They come from altitudes that roughly speaking increase with decreasing frequency. Typical heights observed in the decimetre-to-meter wave band (frequencies $1 \mathrm{GHz}-100 \mathrm{MHz})$ range from the low corona to $(0.5-1) R_{\odot}$

\footnotetext{
*Corresponding author: ludwig.klein@obspm. fr
}

above the photosphere (see reviews by Bastian et al., 1998; Nindos et al., 2008). The radio bursts in this frequency range are in some cases due to gyrosynchrotron radiation, but more often to kinetic plasma processes. They involve non-Maxwellian distributions of energetic electrons and plasma instabilities, produced by electron beams propagating along magnetic field lines that connect the Sun to the Heliosphere or by electron populations trapped in magnetic fields and developing loss-cone distributions. These emissions led to abundant studies of kinetic plasma processes in astrophysics (Melrose, 2017). They are key characteristics of solar activity and a reference for flaring and 
eruptive processes in solar-type stars in general (Crosley \& Osten, 2018). The shape and evolution of the radio spectrum of these bursts are observed by whole Sun radio spectrographs.

In the present effort to understand the relation between the Sun and the Heliosphere, radio astronomy in general and radio spectrographs in particular, are tools to connect in situ observations and radio observations from space, undertaken at frequencies where radio waves cannot travel through the ionosphere of the Earth, to the parent solar activity in the low corona. Groundbased radio observations will enhance the scientific return of the large research endeavour of the 2020s with Parker Solar Probe (NASA) and Solar Orbiter (ESA/NASA). Solar-dedicated instruments ensure optimal coverage with the space missions, which continuously observe the Sun and its disturbances of the heliospheric plasma. This task makes smaller instruments essential for solar-terrestrial physics, in addition to the detailed occasional observations by the large general purpose radio telescopes like VLA (Very Large Array), LOFAR (LOw Frequency ARray), and MWA (Murchison Widefield Array).

Besides their role in solar and solar-heliospheric physics radio spectrographs serve space weather purposes. CMEs and high-energy particles that are sporadically accelerated in the solar corona (especially protons) may cause major disturbances in the Earth's plasma environment, with potential technological consequences and enhanced radiation doses that can extend to the troposphere (e.g., Lanzerotti, 2017, and references therein). Radio bursts are tracers of the triggering and early rise of CMEs before they become visible in the field of view of a space-borne coronagraph, of shock waves in the corona within $1 R_{\odot}$ above the photosphere, and of conditions for the escape of energetic particles from the corona (e.g., Klein, 2021). Radio bursts can also be a space weather nuisance by themselves, with potential effects on GNSS (Global Navigation Satellite System: GPS (Global Positioning System) and other services) (e.g., Carrano et al., 2009; Sato et al., 2019) and air navigation (Marqué et al., 2018).

Several whole Sun radio spectrographs are presently operated by research institutes worldwide (Japan, China, Australia, India, Russia). In the European time zone, radio spectrographs in the metre wave band are operated in Russia (IZMIRAN Moscow, 25-270 MHz), Greece (Thermopylae, 20-650 MHz; Caroubalos et al., 2001), Belgium (Humain radio observatory, 275-1495 MHz), Switzerland (Bleien, 100-5000 MHz). The Astrophysical Institute in Potsdam (Germany) operated a radio spectrograph in the (40-800) $\mathrm{MHz}$ range until 2007 (Mann et al., 1992). The US Air Force operates spectrographs at different sites worldwide within its Radio Solar Telescope Network (RSTN) between 25 and $180 \mathrm{MHz}$. The e-Callisto Network (Benz et al., 2005), which is organised by the former radio astronomy group at the Swiss Federal University of Technology in Zurich (Switzerland), comprises a huge worldwide set of radio spectrographs at $\mathrm{dm}-\mathrm{m}$ wavelengths.

The Nançay radio observatory in central France has a long tradition of hosting instruments that monitor the solar corona at metre wavelengths. The Nançay Radiohoheliograph (NRH; Kerdraon \& Delouis, 1997) produces images at selected frequencies in the $150-450 \mathrm{MHz}$ range, and the Nançay Decameter Array (NDA; Lecacheux, 2000) is a very sensitive spectrograph in the $(20-80) \mathrm{MHz}$ range, observing either solar
Table 1. ORFEES radio spectrograph characteristics.
Type of mounting

Antenna diameter

Polarisation

Geographical longitude

Geographical latitude

Frequency range

Number of sub-bands

Bandwidth of sub-bands

Number of channels

Channel width

Time resolution

Observing time

Data type

Data volume
Equatorial

$5 \mathrm{~m}$

$2 \times$ linear (horizontal and vertical)

$2^{\circ} 11^{\prime} 29.0^{\prime \prime} \mathrm{E}$

$47^{\circ} 22^{\prime} 51.1^{\prime \prime} \mathrm{N}$

(144-1004) $\mathrm{MHz}$

5

$170 \mathrm{MHz}$

2048

$97.65 \mathrm{kHz}$

$100 \mathrm{~ms}$

8-16 UT

FITS

$2.7 \mathrm{~GB} /$ day or jovian radio emission. The need to observe radio spectra in the range where NRH images the Sun, together with the interest of radio burst observations in operational space weather activities, led Paris Observatory and the French Air Force, with their experimental space weather service FEDOME (Fédération des Données Météorologiques de l'Espace) of COSMOS (Centre Opérationnel de Surveillance Militaire des Objets Spatiaux), to devise a new solar radio spectrograph for implementation at Nançay. This instrument, called ORFEES (Observations Radiospectrographiques pour FEDOME et l'Etude des Eruptions Solaires), started operations in 2012. The present article summarises the technical setup of the antenna and receiver (Sect. 2), the data structure (Sect. 3), calibration procedure (Sect. 4), the way to access the data (Sect. 5), and gives some illustrations of observed dynamic spectra (Sect. 6).

\section{The instrument}

\subsection{Overview}

The ORFEES radio spectrograph is located at the Nançay radio observatory in central France. The spectrograph covers the frequency range $144-1004 \mathrm{MHz}$ that is swept ten times per second (total flux density, horizontal and vertical polarization). The radio telescope is operated automatically and tracks the Sun from 8:00 to 16:00 UT every day. The instrument characteristics are summarized in Table 1.

The ORFEES radio spectrograph comprises four parts: the antenna with tracking motor, analog receiver and control system for the analog receiver, the digital radio frequency receiver, and the data acquisition system. Figure 1 shows the whole chain of the radio spectrograph. We describe all components in detail in the following sections.

\subsection{Antenna}

The antenna is an equatorially-mounted parabola with $5 \mathrm{~m}$ diameter, which tracks the Sun. It is controlled manually or automatically by computer. Its position (hour angle and declination) is measured by an analog coder and compared with the coordi- 


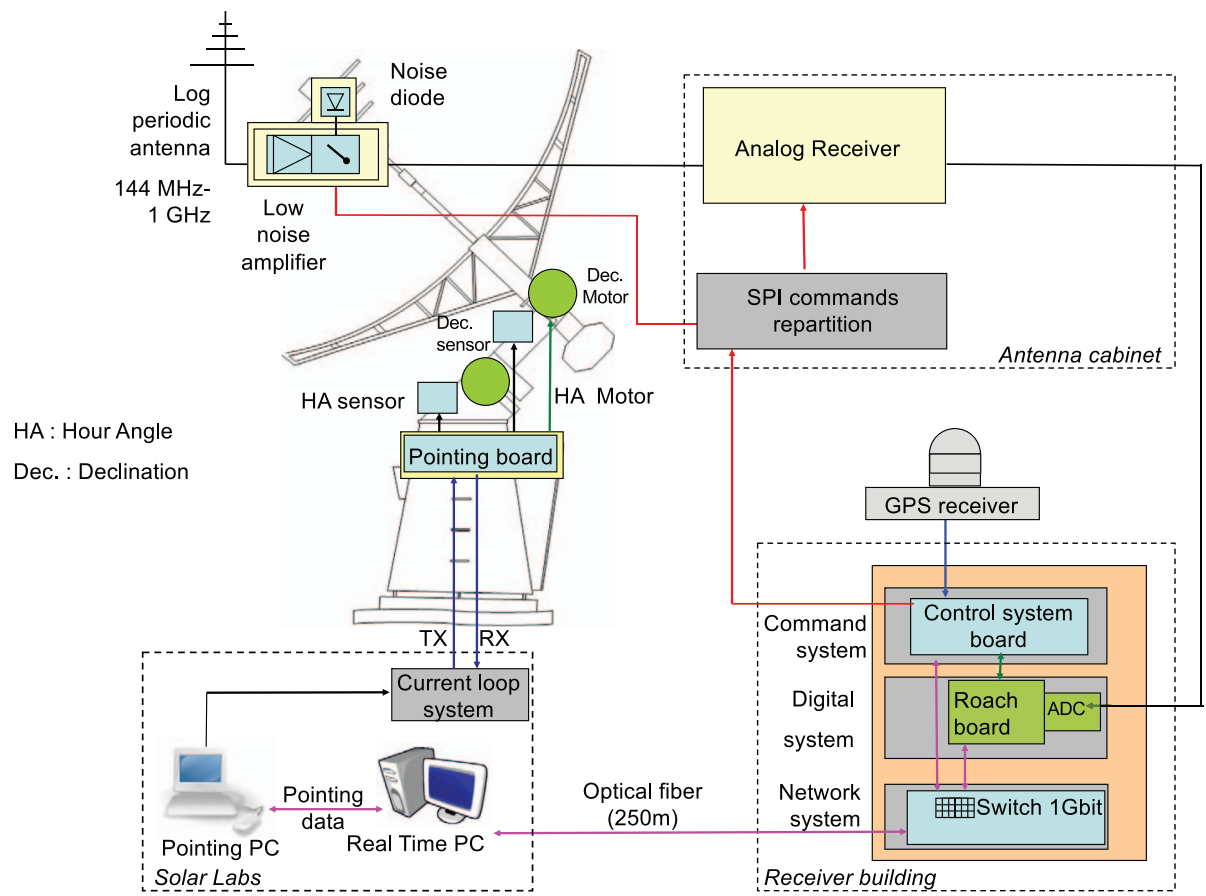

Fig. 1. ORFEES radio spectrograph: antenna, receiver (analog, digital), command system and data acquisition system.

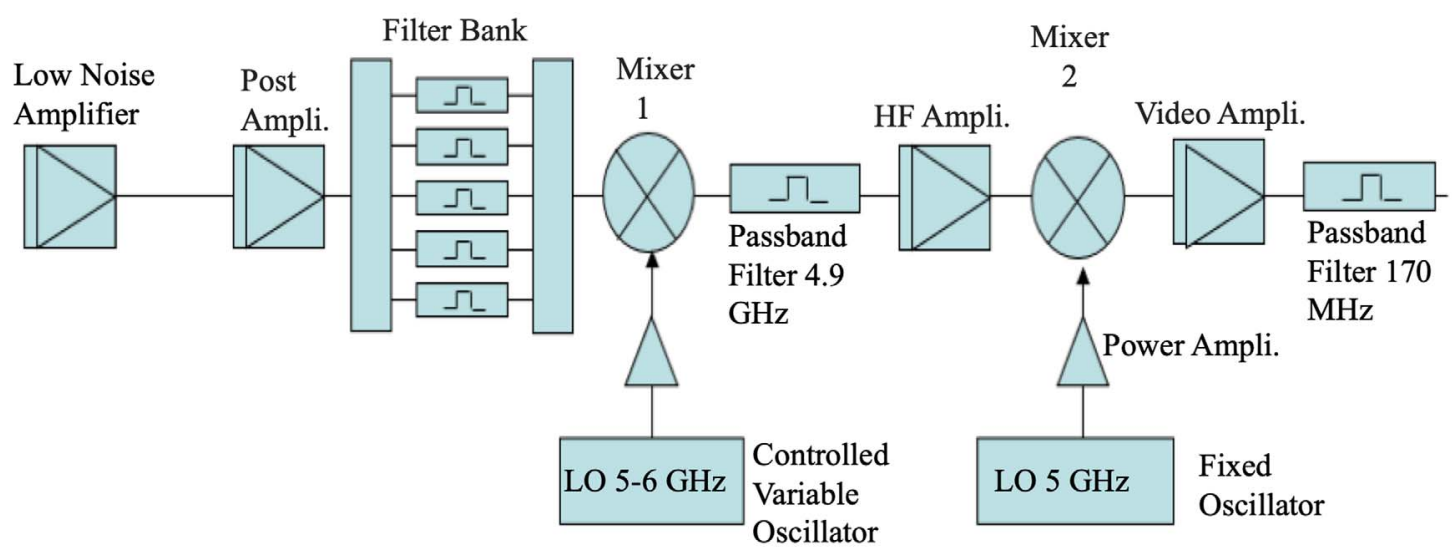

Fig. 2. Block diagram of the ORFEES receiver: superheterodyne receiver.

nates of the radio source. A log-periodic dual-polarization antenna is placed near the focus of the parabola. The antenna provides double linear polarization (vertical and horizontal). The dimensions of the dipoles are adapted to the frequency range from 144 to $1004 \mathrm{MHz}$, but are reduced to minimize the loss of gain as a function of frequency. Low-noise preamplifiers in the antenna front-end serve to avoid intermodulation of strong signals in the reception band. A noise diode in the antenna front-end enables calculation of the reception gain of the whole system, except the preamplifier.

\subsection{Analog receiver}

The receiver is of superheterodyne type, composed of $\mathrm{HF}$ (high frequency) and IF (intermediate frequency) amplifiers, mixers, and detectors. All components are chosen to maximize the dynamic range. The signals from the preamplifier after the log-periodic antenna are transported in two coaxial cables. After filtering, amplification, and heterodyning, the signals emerge from the HF in baseband (between 0 and $200 \mathrm{MHz}$ ) to the digital receiver. Figure 2 shows the block diagram.

The receiver is designed to observe with a high dynamic range, which is affected by terrestrial transmitters. In Nançay the strongest one is high-power digital TV. A filter bank in the first stage of the receiver allows the selection of five sub-bands, which are translated to $4.9 \mathrm{GHz}$ by a mixer and a variable oscillator. The signal is then filtered, amplified and transposed to $100 \mathrm{MHz}$ using a second fixed frequency oscillator. Local oscillators (variable and fixed) are amplified to avoid possible intermodulations in the mixers. A final band-pass filter avoids any possible spectral aliasing. The entire receiver is 


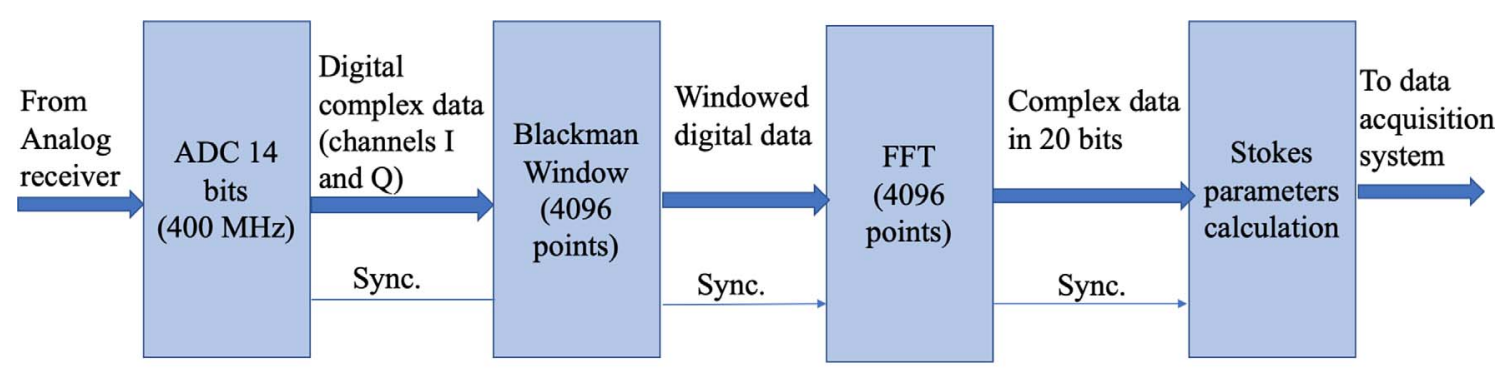

Sync. : Synchronisation signal

$\mathrm{I}$ : bus represents the I channel of the ADC card (real data)

$\mathrm{Q}$ : bus represents the $\mathrm{Q}$ channel of the $\mathrm{ADC}$ card (imaginary data)

Fig. 3. Block diagram of the ORFEES digital receiver.

controlled by an 8-bit ATMEGA micro-controller. ${ }^{1}$ The system is synchronized and timestamped with a GPS. ${ }^{2}$

\subsection{Digital receiver}

The signals from the analog receiver are converted by analog-to-digital converters (ADCs) with a resolution of 14 bits. The outputs of the ADCs have complex values (real $(I)$ and imaginary $(Q)$ data). These ADCs are connected to the $\mathrm{ROACH}$ (Reconfigurable Open Architecture Computing Hardware) ${ }^{3}$ signal processing card. The sampling frequency of $400 \mathrm{MHz}$ allows us to treat RF bands ranging from 0 to $200 \mathrm{MHz}$. For this reason, we use five bands to cover the frequency range of ORFEES. The minimum processing time needed for each band is $20 \mathrm{~ms}$.

The truncation of the signal by the ADC is equivalent to multiplying the time history of the signal by a rectangular window. The resulting Fourier transform will therefore be convolved with a sinc function. But since the spectral characteristics of such a window are not adapted to the spectral analysis of the signal, another window function is needed. A Blackman-type time window is applied to improve the spectral aspect of the digital signal. The Fast Fourier Transform (FFT) with 4096 points is then calculated to provide spectra in 2048 channels with a frequency resolution of $97.65 \mathrm{kHz}$. Thereafter, the Stokes parameters $I$ (total intensity) and $V$ (circularly polarized intensity) are calculated for each channel. Figure 3 summarizes all components of the ORFEES digital receiver.

\section{Processing and description of the data}

The raw data from the ROACH board are sent to the data acquisition system. This system initializes the control system for each observation, calibrates and processes raw spectra, and records the final spectra in FITS format. A header is created to trace any error and identify the band number associated with the spectrum. The calculated spectra are dated by GPS. The data processing is performed by the data acquisition system in real

\footnotetext{
${ }^{1}$ http://ww1.microchip.com/downloads/en/DeviceDoc/doc2503. pdf

${ }^{2}$ https://static.garmincdn.com/pumac/GPS_18x_Tech_Specs.pdf

${ }^{3}$ https://casper.ssl.berkeley.edu
}

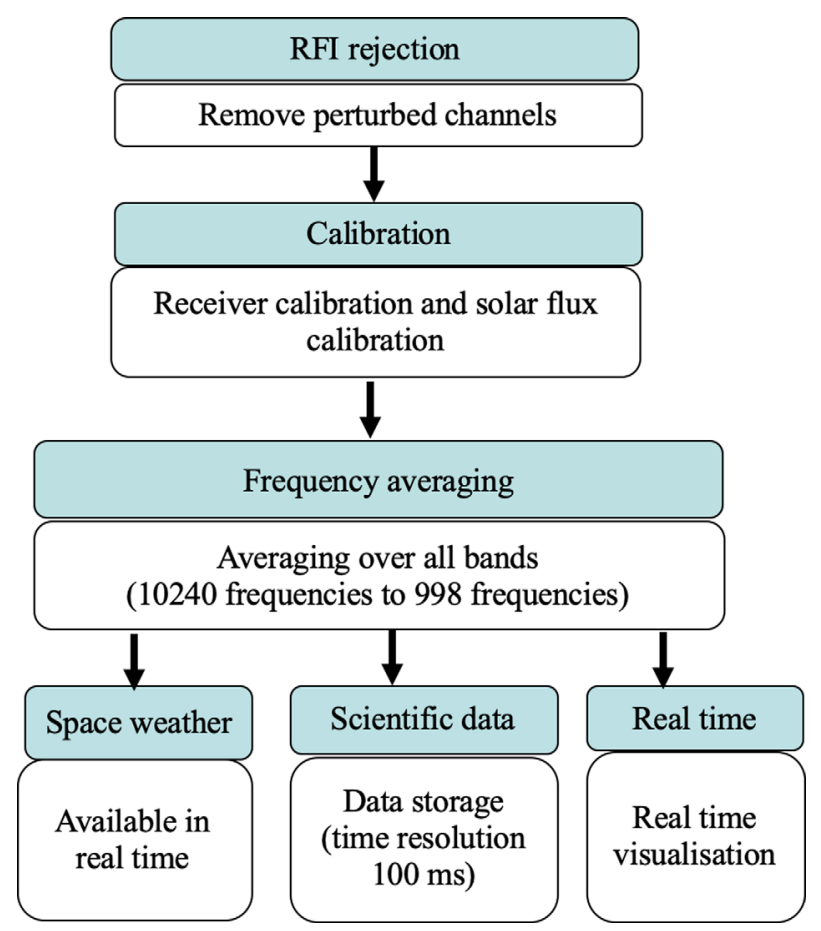

Fig. 4. Organizational chart summarising data processing in the ORFEES data acquisition system.

time. Figure 4 summarizes the steps of the data processing up to the acquisition in real-time.

The first operation in the data acquisition system is to reject the RFI (radio-frequency interference) at pre-defined frequencies within the ORFEES band. RFI is a recurring problem in radio astronomy receivers, and a number of emitters are detected at Nançay. These signals are very strong compared to the solar emission. We adopted the solution to suppress perturbed frequencies. Table 2 summarizes the RFI detected in the ORFEES band. ${ }^{4}$ This table is updated annually on the RSDB website. $^{5}$

\footnotetext{
${ }^{4}$ https://www.anfr.fr/fileadmin/mediatheque/documents/tnrbf/

TNRBF_2020-03-16.pdf

${ }^{5}$ https://rsdb.obs-nancay.fr/\#!/documentation
} 
Table 2. Radio frequency interference in the ORFEES frequency range and its users: 4G/LTE (Long Term Evolution), DVB-T (Digital Video Broadcasting Terrestrial), GSM (Global System for Mobile Communications).

\begin{tabular}{lccc}
\hline $\begin{array}{l}\text { Band } \\
\text { number }\end{array}$ & $\begin{array}{c}\text { Start } \\
\text { frequency }\end{array}$ & $\begin{array}{c}\text { End } \\
\text { frequency }\end{array}$ & $\begin{array}{c}\text { Used } \\
\text { by }\end{array}$ \\
\hline 1 & 148 & 149.90 & $\begin{array}{c}\text { Space communications } \\
\text { Radio diffusion }\end{array}$ \\
1 & 175 & 176 & Radio diffusion \\
1 & 245 & 270 & - \\
2 & 360 & 380 & - \\
2 & 391 & 393 & TV (DVB-T) \\
2 & 471.25 & 477.75 & TV (DVB-T) \\
3 & 495.25 & 501.75 & TV (DVB-T) \\
3 & 519.25 & 525.75 & TV (DVB-T) \\
3 & 591.25 & 597.75 & TV (DVB-T) \\
3 & 631.25 & 637.75 & TV (DVB-T) \\
3 & 647.25 & 653.75 & Mobile telephony \\
4 & 663.25 & 669.75 & 4G/LTE \\
4 & 775.25 & 781.75 & 4G/LTE \\
4 & 783.25 & 789.75 & 4G/LTE \\
4 & 791.25 & 797.75 & 4G/LTE \\
4 & 799.25 & 805.75 & 4G/LTE \\
4 & 807.25 & 813.75 & 4G/LTE \\
4 & 815.25 & 821.75 & 4G/LTE \\
4 & 823.25 & 829.75 & GSM \\
5 & 890 & 904 & GSM \\
5 & 925 & 960 &
\end{tabular}

Since the characteristics of the receiver vary across the frequency band, a gain correction is established. The correction table is obtained by calibration observations as described in Section 4. Thereafter, to reduce noise, the channels are averaged around fixed frequencies. The number of averaged channels and the final frequencies are defined in a specific table. Four neighboring frequency channels are averaged in the first band, eight in the second and third band, and 16 in the fourth and fifth band. Every $100 \mathrm{~ms}$, the entire frequency range is sampled at 10,240 frequencies, which are reduced to 998 after integration and suppression of frequencies affected by RFI. The spectra are then integrated again for real-time visualization. In the end, the data is converted into an appropriate file type (binary format, space weather format, and scientific FITS with interference rejection).

The scientific data in FITS format is organized in three extensions, as shown in Figure 5. The primary extension contains the header with all information about data and the instrument. The second extension contains the frequency tables in the five sub-bands. Table 3 summarises the start and the end frequency of each sub-band and the number of frequencies. The last extension contains the time array (milli-seconds of the day of the start of the interval) and the flux densities (Stokes parameters I and V) in the five sub-bands.

\section{Calibration}

The calibration has to convert the measurements into flux density, expressed in solar flux units $(1 \mathrm{sfu}=$ $\left.10^{-22} \mathrm{~W} \mathrm{~m} \mathrm{~m}^{-2} \mathrm{~Hz}^{-1}\right)$. The signal at the output of a solar radio spectrograph $P_{r x}$ is the sum of the solar emission $F_{\text {solar }}$, the galactic background $F_{\text {galac }}$ and the system noise of the instrument $G_{r x}$ :

$$
P_{r x}=G_{r x}+F_{\text {solar }}+F_{\text {galac }} .
$$

The calibration process proceeds in two steps, receiver calibration and flux calibration. The main aim of the receiver calibration is to convert the receiver data (arbitrary unit) to antenna temperature values. In this step, we calculate the coefficients related to the entire receiver chain. A noise diode calibration system is used to calculate the conversion coefficients at each frequency. The coefficients of receiver calibration are $C_{r c}=$ $T_{d} / D_{r x}$, where $D_{r x}$ is the receiver value and $T_{d}$ is the temperature of the noise diode. The coefficients are updated regularly, depending on the state of the system, but at least four times a year.

The aim of the flux density calibration is to calculate coefficients that convert antenna temperatures to flux densities in solar flux units. This is done with respect to the quiet Sun, the only celestial reference source strong enough to be observed above background by a $5 \mathrm{~m}$ dish. Elgaroy et al. (1981) proposed an absolute calibration of solar flux in the dm-metric range using the simultaneous calibration of various solar radio instruments at times when the solar flux was stable. For ORFEES we use an analytical formulation of the spectrum of the quiet Sun. Benz (2009) compiled measurements of the flux density between $30 \mathrm{MHz}$ and $300 \mathrm{GHz}$ made in 1964 and 1976, which were the minima of solar cycles 20 and 21 , respectively, and represented them by piecewise power laws. The spectra in the frequency range of ORFEES are $(f$ is the frequency in $\mathrm{MHz}$ ):

$$
\begin{gathered}
F_{\text {solar }}=1.94 \times 10^{-4} \times f^{1.992}[\mathrm{sfu}](30-350 \mathrm{MHz}) \\
F_{\text {solar }}=8.45 \times 10^{-1} \times f^{0.5617}[\mathrm{sfu}](350-6000 \mathrm{MHz}) .
\end{gathered}
$$

The flux density at solar maximum is higher. Since ORFEES started operations near solar maximum, the above spectra were scaled by flux density measurements with the Radio Solar Telescope Network.

The RSTN (San Vito) radio spectrograph has three frequencies in the range of ORFEES $(245,408,610 \mathrm{MHz})$. Average values of measurements in February 2015 yield the combined model currently used to calibrate ORFEES data:

$$
\begin{gathered}
F_{\text {solar }}=3.29 \times 10^{-4} \times f^{1.992}[\mathrm{sfu}](30-350 \mathrm{MHz}) \\
F_{\text {solar }}=14.15 \times 10^{-1} \times f^{0.5617}[\mathrm{sfu}](350-1004 \mathrm{MHz}) .
\end{gathered}
$$

These conversion coefficients need to be adapted by the user if a reliable flux calibration is required. Quiet-Sun flux densities at $1000 \mathrm{MHz}$ may indeed vary by more than a factor 3 over the solar cycle (Fig. 2 of Dudok de Wit et al., 2014). ORFEES data can be re-calibrated by the user. The daily records start well before the antenna points to the Sun and end after the Sun leaves the antenna beam. Hence, the early and late records on each day show the sum of the flux of the galactic background and the system noise of the instrument. 


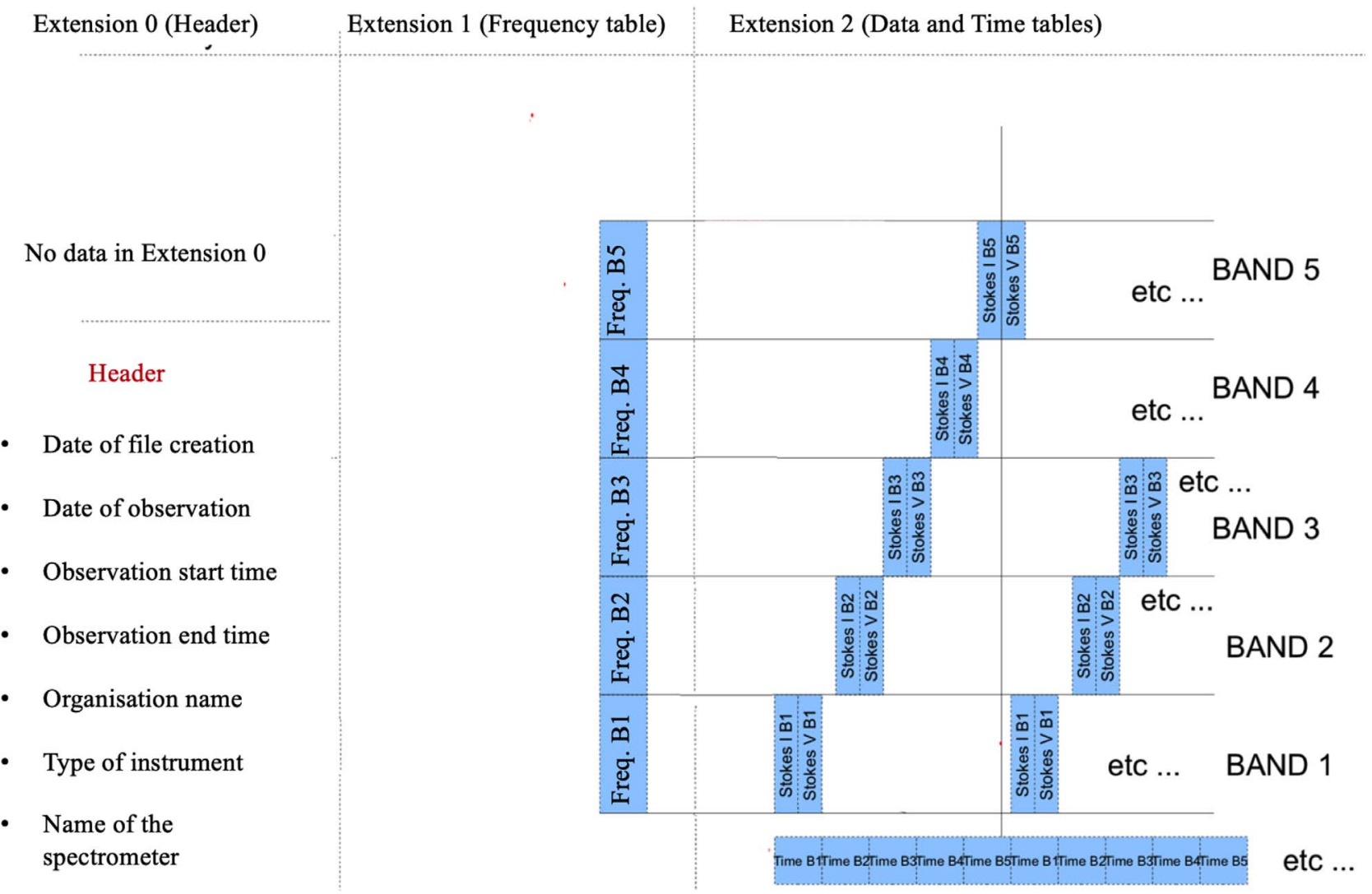

Fig. 5. ORFEES FITS data format description.

Table 3. ORFEES sub-bands with the frequency range and the number of frequencies for each sub-band.

\begin{tabular}{lccc}
\hline $\begin{array}{l}\text { Sub-band } \\
\text { number }\end{array}$ & $\begin{array}{c}\text { Start frequency } \\
(\mathrm{MHz})\end{array}$ & $\begin{array}{c}\text { End frequency } \\
(\mathrm{MHz})\end{array}$ & $\begin{array}{c}\text { Number of } \\
\text { frequencies }\end{array}$ \\
\hline 1 & 144.13 & 315.13 & 431 \\
2 & 315.60 & 484.93 & 215 \\
3 & 485.60 & 654.93 & 164 \\
4 & 656.67 & 823.86 & 86 \\
5 & 826.67 & 1004.7 & 102 \\
\hline
\end{tabular}

\section{Data and software distribution}

The routines to read ORFEES data with IDL and python are available in the Nançay radio solar database. ${ }^{6}$ In addition, the user can use the Spectrum_gui software to plot ORFEES data. Spectrum_gui ${ }^{7}$ is a widget-based procedure developed by the computer science team at Paris Observatory/LESIA in the IDL language. The main function of this widget is to plot any kind of solar dynamic radio spectrum with several options such as 1D cuts along the frequency and time axes or different methods to compute the background.

The ORFEES real-time system produces several types of data, which are written into files that grow during the observation:

\footnotetext{
${ }^{6}$ https://rsdb.obs-nancay.fr/\#!/documentation

${ }^{7}$ http://secchirh.obspm.fr/spip.php?article44
}

- Raw data files: the data at 2048 frequencies in each of the five sub-bands are written into a succession of files of length $2.8 \mathrm{~GB}$, corresponding to one hour of observation. These files are deleted after a few days.

- Scientific data file: this file in FITS format contains the data that will remain available for scientific exploitation. The size is around $2.7 \mathrm{~GB}$ for eight hours of observations. The description of this file is provided in Section 3.

- Space weather data file: A synthesized data set with a reduced amount of data is provided to the FEDOME space weather center. These data show the dynamic spectra observed by ORFEES and NDA, integrated over one second, and the time evolution of the radio sources at selected frequencies observed by the Nançay Radioheliograph. They are transmitted to FEDOME in near real-time (one minute cadence) as a plot, in the same format as the plots on the Radio Monitoring website. The data file is sent at the end of the day.

In addition, a real-time visualization is freely available on the Nançay station website. ${ }^{8}$ It shows the last hour of observations and is updated every minute. The scientific data file with the full resolution is available one day after observation on the radio solar database. ${ }^{9}$

\footnotetext{
${ }^{8}$ https://realtime.obs-nancay.fr/orfees/

${ }^{9}$ https://rsdb.obs-nancay.fr
} 

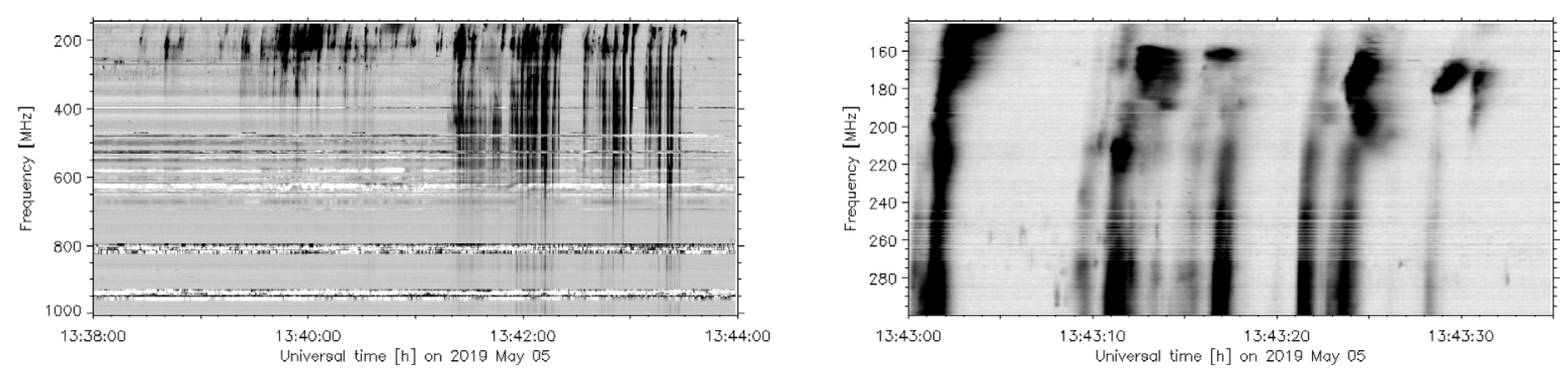

Fig. 6. Dynamic spectra of a group of type III bursts on 2019 May 05, showing the entire group (left panel) and individual bursts (right panel) with $0.1 \mathrm{~s}$ resolution.

\section{Examples of solar observations}

In this section we illustrate some ORFEES observations of typical solar radio bursts and the Spectrum_gui software.

\subsection{Type III bursts and narrowband spikes}

Figure 6 shows the dynamic spectrum of a group of type III bursts, which occurred during the rise of a soft X-ray burst (GOES; peak B9.8 at 13:42 UT; NOAA Space Weather Prediction $C^{-n t e r}{ }^{10}$ ). The bursts are grouped in different clusters with higher start frequencies after 13:41 UT. The right panel shows the last $40 \mathrm{~s}$ of the former plot focussing on the (144-300) $\mathrm{MHz}$ band. The type III bursts have spectral fine structure in that range: narrow-band brightenings are observed near the low-frequency cutoff of the burst at 13:43:17 UT (170-185 $\mathrm{MHz}$ ) and throughout the bursts between 13:43:10 and 13:43:18 UT; a bidirectional burst (Sect. 3.1 of Nindos et al., 2008, and references therein) drifts towards lower and higher frequencies, starting at $180 \mathrm{MHz}$ at 13:43:24 UT.

The spectra show the flux density difference with respect to a pre-event scan. The horizontal bands are frequencies disturbed by artificial radio emitters. The spectrum above $450 \mathrm{MHz}$ is heavily affected by them, and a comparison between this relatively recent observation and earlier data in the following figures shows that the situation is rapidly worsening.

The increase of mobile network users is an important fact that led the telephone companies to extend beyond the historically allocated frequencies (around $900 \mathrm{MHz}$ ). The frequency range above $650 \mathrm{MHz}$ was recently allocated to telephone companies. These frequencies are used for 4G/LTE (Long term evolution) communications. All frequencies in the ORFEES range occupied by artificial radio emitters are listed in Table 2.

The radio burst in the top left panel of Figure 7 occurred during the rise of a soft X-ray burst (GOES; peak C4.4 at 08:23 UT; NOAA Space Weather Prediction Center, see Footnote 10). It was accompanied by a microwave burst observed by the San Vito station of the Radio Solar Telescope Network of the US Air Force (RSTN, ${ }^{11}$ not shown here). Its brightest phase occurred between 8:12:30 and 8:13:20, with a spectrum that decreased from 1.415 to $15.4 \mathrm{GHz}$. The ORFEES spectrum shows a diffuse emission in its high-frequency part at that time, which by comparison with the microwave records

\footnotetext{
10 https://www.solarmonitor.org/

11 https://www.ngdc.noaa.gov/stp/space-weather/solar-data/solarfeatures/solar-radio/rstn-1-second/
}

may be the low-frequency extension of a gyrosynchrotron spectrum. The time histories at 300 and $900 \mathrm{MHz}$, respectively, plotted in the top and bottom-right panels of Figure 7, show clearly that the emission observed by ORFEES has two components: a broadband continuum, which evolves on a time scale of about ten seconds, is probably the low-frequency extension of the gyrosynchrotron spectrum. On top of the continuum numerous short (sub-second time scale) bursts are observed.

The dynamic spectrum shows the short bursts as narrowband spikes (Benz, 2008; Nindos et al., 2008, Sect. 5.1, and references therein), seen by ORFEES between its high-frequency border at $1000 \mathrm{MHz}$ and $200 \mathrm{MHz}$. This becomes clearer after subtracting the background continuum emission by a sliding median filter in the bottom left panel of Figure 7. At low frequencies the spikes were visible before the continuum, with a well-defined highfrequency cutoff between 500 and $600 \mathrm{MHz}$. The cluster extended to higher frequencies at the start and then receded to the previous lower frequencies at the end of the most intense continuum emission. This suggests a physical relationship between the spike emission and the acceleration of the electrons emitting the presumable gyrosynchrotron continuum. It has been argued in the literature that narrowband decimetric spikes might be elementary signatures of electron acceleration, but this is still subject to debate (see Benz, 2008, and references therein).

\subsection{Type II burst}

Figure 8 displays the dynamic spectrum of a type II burst in the top panel. The event was presented in Section 5.2 of Cairns et al. (2020). The emission starts at unusually high frequencies. It has one broad band with a complex internal structure that starts above $700 \mathrm{MHz}$, and a second, narrower band starting a little later near $350 \mathrm{MHz}$. Both bands have complex fine structures. The low-frequency band, displayed in more detail in the bottom left figure between 150 and $400 \mathrm{MHz}$, comprises two separate lanes, usually referred to as split bands. The split bands change in the course of the event. They are themselves composed of short bursts with narrow bandwidth. The brightest lanes are seen at the high-frequency edge of the fundamental band. A particularly bright lane stops abruptly at 12:03:46 UT near $200 \mathrm{MHz}$.

The figure on the bottom right also shows the harmonic band in the same time interval as the left figure. The frequency axis is from 300 to $800 \mathrm{MHz}$, i.e., twice the values of the ordinate in the left figure. Therefore identical features in the fundamental and the harmonic band will be at the same relative position on the vertical axes in the two figures. 

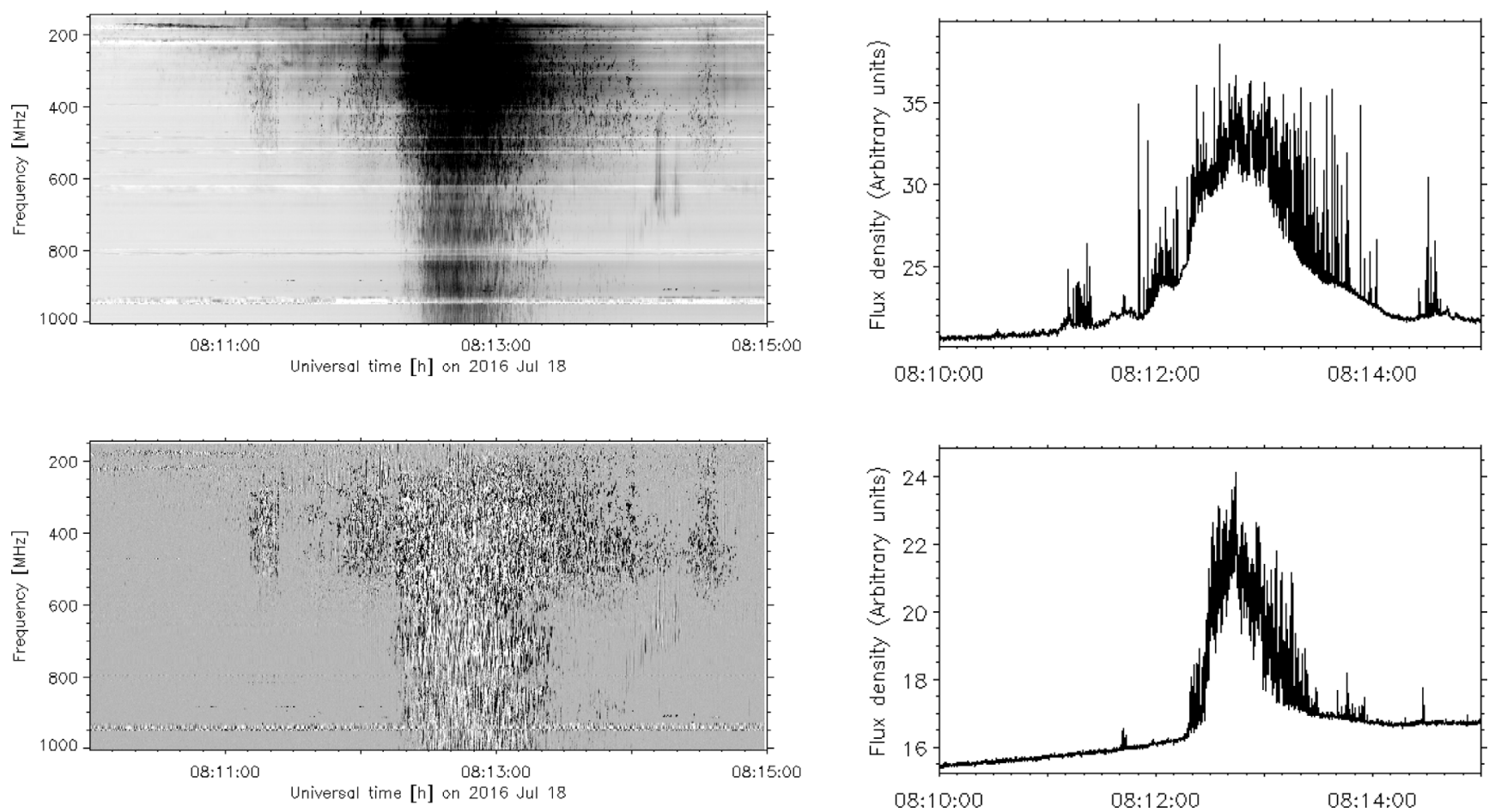

Fig. 7. Dynamic spectra on 2016 Jul 18 showing a group of spike bursts on top of a continuum (left column: raw spectrum in the top panel, spectrum after subtraction of a median filter over $2 \mathrm{~s}$ in the bottom panel). The time histories in the right panels are at 300 (top) and $900 \mathrm{MHz}$, respectively.
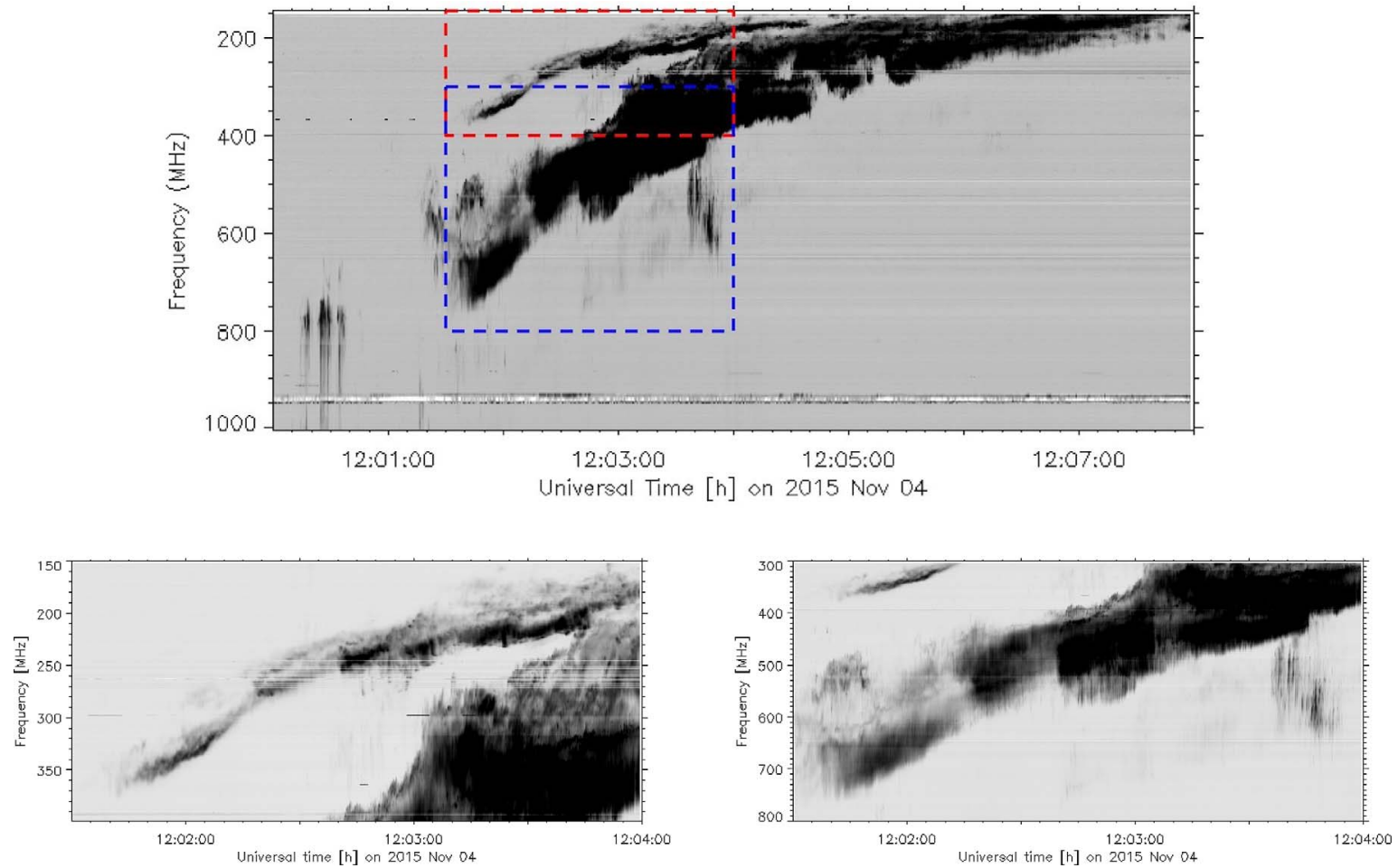

Fig. 8. Top: Dynamic spectrum of a type II burst on 2015 Nov 04 from 144 to $1000 \mathrm{MHz}$, showing fundamental and harmonic bands. The overlaid rectangles delimit parts of the fundamental (red) and harmonic (blue) band. Bottom: Dynamic spectra in the ranges delimited by the red (left panel) and blue (right panel) rectangles in the top panel. The frequency axes of the two plots have a ratio of 2:1. A reference profile (mean from 12:00 to 12:01 UT) has been subtracted. 

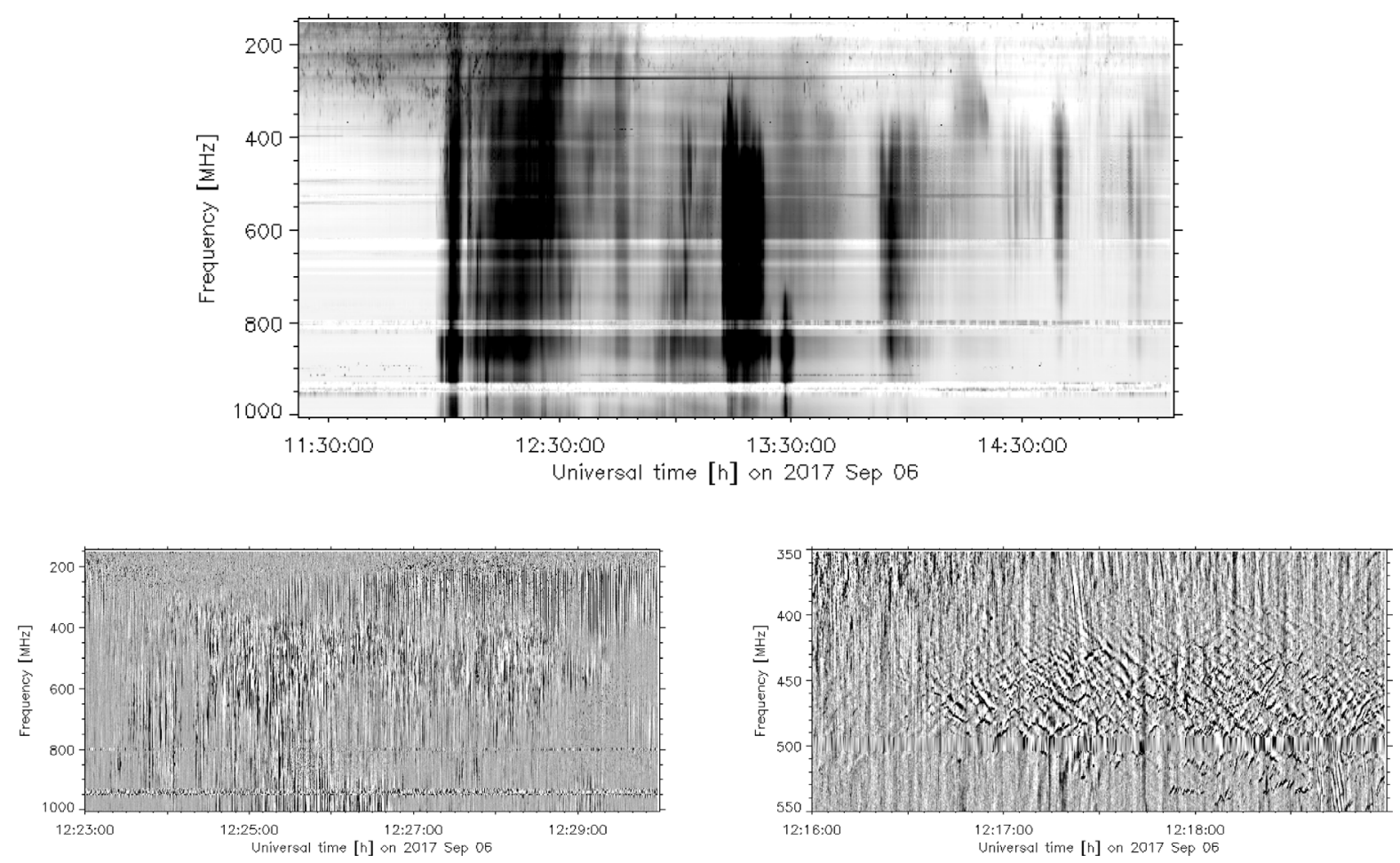

Fig. 9. Dynamic spectrum of a type IV burst on 2017 September 06. Top panel: the entire event. Bottom panels: two episodes of fine structure (a sliding median was subtracted over $3 \mathrm{~s}$ and $10 \mathrm{~s}$, respectively).

The two bands have similar morphology. The harmonic is split into two bands, as the fundamental, but the low-frequency split band is weaker in the fundamental. The relative weakness of the low-frequency split band of the fundamental becomes very clear after 12:03, when the low-frequency split band of the harmonic is bright at $300 \mathrm{MHz}$, without a clear counterpart in the fundamental near $150 \mathrm{MHz}$. This may be due to the freefree absorption of the fundamental split band. Similarities are more prominent in the high-frequency split band, for instance, the abrupt stop or interruption at 12:03:46 UT near 200 and $400 \mathrm{MHz}$, respectively. Thereafter the gap between the fundamental and the harmonic band is filled by herringbone bursts that emanate from the harmonic since 12:03:40. They are, if at all, less clearly visible in the fundamental band.

\subsection{Type IV burst}

During a late period of activity, the strongest solar soft X-ray burst (X9.3, peak at 12:02 UT) in activity cycle 24 occurred on 2017 Sep 06. At radio wavelengths, it was accompanied by a type IV burst starting near 12 UT. The dynamic spectrum observed by ORFEES is plotted in the top panel of Figure 9. The emission covered the entire band of ORFEES. The emission seen before 12:00 UT below $400 \mathrm{MHz}$ is part of a noise storm, which is not related to the flare. This noise storm continued throughout the time interval displayed in the figure but with reduced flux density. It is composed of a broadband continuum and superposed narrowband type I bursts. The white band below $200 \mathrm{MHz}$ after 12:50 UT is an artifact due to the subtraction of a spectral scan at 14:45:46 UT, where the low-frequency emission still had a weak, but significant, contribution from the noise storm.
The type IV burst is a broadband continuum with pronounced temporal and spectral fine structures. The dynamic spectrum in the top panel is displayed with a $1 \mathrm{~s}$ time resolution. The two bottom panels illustrate spectral fine structure observed with $0.1 \mathrm{~s}$ integration time. A sliding median filter in time over $3 \mathrm{~s}$ (left panel) and $10 \mathrm{~s}$ (right panel) was subtracted. The left panel shows mainly broadband pulsations, with a more complex structure at the higher frequencies. The right panel shows broadband pulsations, with superposed fiber bursts or zebra pattern (Sect. 5.3 of Nindos et al., 2008, and references therein) after 12:16:30 UT.

\section{Summary}

The ORFEES radio spectrograph has been operating at the Nançay station since 2012, with daily observations 8-16 UT. The number of days per year where observations were acquired, albeit not always over the full 8-16 UT range, varied between 329 and 353. ORFEES is hence in a phase of regular data production for the scientific community worldwide. The instrument supports the scientific investigations with Solar Orbiter and Parker Solar Probe, as illustrated by the study of the 2020 Nov 29 solar energetic particle event (Kollhoff et al., 2021). ORFEES complements the spectrographs in Bleien (Switzerland), Humain (Belgium), and Thermopylae (Greece), operating at similar longitudes, with high time resolution and sensitivity, at a site where disturbances by artificial radio emitters are for the time being still manageable. Together with the Nançay Decametre Array, it covers the frequency band from $1 \mathrm{GHz}$ to near the ionospheric cutoff and therefore allows nearly seamless coverage of the decimetre-meter wave spectrum out to the 
domain of space borne radio spectrographs. The band where ORFEES observes dynamic spectra covers the range where NRH takes images. The data are open to being used without restriction for research in solar-terrestrial physics and space weather applications. The access to the data of all solar radio observations conducted in Nançay has recently been facilitated by a common database.

The data and the operational mode of ORFEES make the instrument suitable for space weather applications. These applications motivated the French Air Force to support the project. The experimental space weather center FEDOME is provided with real-time overview data from ORFEES and the other solar instruments at Nançay Observatory. As an academic institution Paris Observatory guarantees the operations on a best-effort basis. To within this limitation, ORFEES is open to cooperation with other actors in the space weather domain. The ORFEES concept could be integrated into a worldwide network of monitoring instruments for scientific use or space weather services. The space weather utilization needs an implication by stakeholders, which in Europe are the European Union (EU) and the European Space Agency (ESA).

Acknowledgements. The ORFEES spectrograph has benefitted from financial support by the Air Force as part of the FEDOME innovation project, the Agence Nationale pour la Recherche (ANR/ASTRID, DGA) project Outils radioastronomiques pour la météorologie de l'espace (ORME, contract No. ANR-14-ASTR-0027) and by the Programme National Soleil-Terre (PNST) of CNRS/INSU. The authors are indebted to the referees for their careful reading and very helpful comments. The editor thanks two anonymous reviewers for their assistance in evaluating this paper.

\section{References}

Bastian TS, Benz AO, Gary DE. 1998. Radio emission from solar flares. Ann. Rev. Astron. Astrophys. 36: 131-188.

Benz AO. 2008. Flare observations. Living Rev Sol Phys 5: 1. https://doi.org/10.12942/lrsp-2008-1.

Benz AO. 2009. Radio emission of the quiet sun. Landolt Börnstein 4B: 103. https://doi.org/10.1007/978-3-540-88055-4_5.

Benz AO, Monstein C, Meyer H. 2005. Callisto - a new concept for solar radio spectrometers. Sol Phys 226(1): 143-151. https://doi. org/10.1007/s11207-005-5688-9.

Cairns IH, Kozarev KA, Nitta NV, Agueda N, Battarbee M, et al. 2020. Comprehensive characterization of solar eruptions with remote and in-situ observations, and modeling: The major solar events on 4 November 2015. Sol Phys 295(2): 32. https://doi.org/ 10.1007/s11207-020-1591-7.

Caroubalos C, Maroulis D, Patavalis N, Bougeret J, Dumas G, et al. 2001. The new multichannel radiospectrograph ARTEMIS-IV/ HECATE, of the University of Athens. Exp Astron 11: 23-32.
Carrano CS, Bridgwood CT, Groves KM. 2009. Impacts of the December 2006 solar radio bursts on the performance of GPS. Radio Sci 44: RS0A25. https://doi.org/10.1029/2008RS004071.

Crosley MK, Osten RA. 2018. Low-frequency radio transients on the active M-dwarf EQ Peg and the search for coronal mass ejections. Astrophys J 862(2): 113. https://doi.org/10.3847/15384357/aacf02.

Dudok de Wit T, Bruinsma S, Shibasaki K. 2014. Synoptic radio observations as proxies for upper atmosphere modelling. J Space Weather Space Clim 4: A06. https://doi.org/10.1051/swsc/ 2014003.

Elgaroy O, Slottje C, Tlamicha A, Urbarz H, Zanelli C, Zlobec P, Bougeret J, Kerdraon A, Noe J. 1981. Simultaneous calibration of solar radio instruments from decimetre to decametre wavelengths. Astron Astrophys Suppl Ser 44: 165.

Kerdraon A, Delouis J-M. 1997. The Nançay Radioheliograph. In: Coronal physics from radio and space observations, Trottet $\mathrm{G}$ (Eds.), Vol. 483 of Lecture Notes in Physics, Springer, Berlin, Heidelberg, New York, pp. 192-201.

Klein K-L. 2021. Radio astronomical tools for the study of solar energetic particles I. Correlations and diagnostics of impulsive acceleration and particle propagation. Front Astron Space Sci 7: 105. https://doi.org/10.3389/fspas.2020.580436.

Kollhoff A, Kouloumvakos A, Lario D, Dresing N, GómezHerrero R, et al. 2021. The first widespread solar energetic particle event observed by Solar Orbiter on 2020 November 29. $A \& A$, in press. https://doi.org/10.1051/0004-6361/202140937.

Lanzerotti LJ. 2017. Space weather: historical and contemporary perspectives. Space Sci Rev 212: 1253-1270. https://doi.org/ 10.1007/s11214-017-0408-y.

Lecacheux A. 2000. The Nançay Decameter Array: A useful step towards giant, new generation radio telescopes for long wavelength radio astronomy. In: Radio astronomy at long wavelengths, Stone R., Weiler K., Goldstein M., Bougeret J.-L. (Eds.), Vol. 119 of AGU Monograph, pp. 321-328.

Mann G, Aurass H., Voigt W., Paschke J.. 1992. Preliminary observations of solar type II bursts with the new radiospectrograph in Tremsdorf. In: Coronal streamers, coronal loops, and coronal and solar wind composition, Mattok C. (Ed.), Vol. 348 of ESA Special Publication, pp. 129-132.

Marqué C, Klein K-L, Monstein C, Opgenoorth H, Pulkkinen A, Buchert S, Krucker S, Van Hoof R, Thulesen P. 2018. Solar radio emission as a disturbance of aeronautical radionavigation. J Space Weather Space Clim 8: A42. https://doi.org/10.1051/swsc/ 2018029.

Melrose DB. 2017. Coherent emission mechanisms in astrophysical plasmas. Rev Mod Plasma Phys 1: 5. https://doi.org/10.1007/ s41614-017-0007-0.

Nindos A, Aurass H, Klein K-L, Trottet G. 2008. Radio emission of flares and coronal mass ejections. Sol Phys 253: 3-41. https://doi. org/10.1007/s11207-008-9258-9.

Sato H, Jakowski N, Berdermann J, Jiricka K, Heßelbarth A, Banyś D, Wilken V. 2019. Solar radio burst events on 6 September 2017 and its impact on GNSS signal frequencies. Space Weather 17(6): 816-826. https://doi.org/10.1029/2019SW002198.

Cite this article as: Hamini A, Auxepaules G, Birée L, Kenfack G, Kerdraon A, et al. 2021. ORFEES - a radio spectrograph for the study of solar radio bursts and space weather applications. J. Space Weather Space Clim. 11, 57. https://doi.org/10.1051/swsc/2021039. 